\title{
New test reveals early diapause in pink bollworm
}

\author{
Thomas A. Miller $\square$ Mohamed Salama $\square$ Richard C. Weddle $\square$ Saku Sivasupramaniam
}

\begin{abstract}
Through basic research into genetic control of pink bollworm development, we devised a rapid immunological method of determining diapause in pink bollworm larvae - formerly a monthlong process which now requires only 24 hours. This new "ELISA" method has revealed that the pink bollworm larvae in the Imperial Valley sometimes enter diapause weeks ahead of the expected September date for diapause induction. The earliness of diapause induction is correlated with the severity of stress on the cotton plant caused by whitefly infestations.
\end{abstract}

The pink bollworm, Pectinophora gossypiella, is thought to have evolved in Australia where the only other two species of the same genus, $P$. endema and $P$. scutigera, are also found. Of these three species, only the pink bollworm, a worldwide pest of cotton, has the ability to enter diapause, an arrested state of development akin to vertebrate hibernation in which fully mature larvae are able to survive host-free periods. Indeed, the pink bollworm is thought to have spread originally when cotton seeds contaminated with diapausing larvae were shipped from India. Eventually, they made their way to virtually all of the world's other cotton-growing areas.

Because short day-length and cooler temperatures of early fall induce diapause, a successful strategy has been to defoliate cotton in advance of the pink bollworm diapause period, around September 1 each year in the United States (fig. 1). Since this "short season" strategy was introduced in 1989 , it has drastically reduced populations in the Imperial Valley.

Traditionally, measuring diapause involves collecting cotton bolls from the field in fall and holding the bolls under ambient conditions for several days. In bolls heavily infested with pink boll- worms, mature fourth-instar larvae begin leaving and searching for pupation or diapause sites. These wandering larvae are harvested, placed in Petri dishes under paper towels and left to "incubate" in room temperature conditions. Any larvae remaining after 30 days in the Petri dishes are considered in diapause, the remainder having pupated.

This older method's drawback is that the percentage of larvae actually entering diapause depends on conditions during incubation. Thus, the actual percentage of diapause determined with these laboratory procedures, especially after 1 month, may differ from what actually occurs in the field.

A new method of determining diapause in pink bollworm is based on characteristic changes in hemolymph that occur only in its diapausing larvae. It has been known for many years that diapausing insects produce characteristic proteins corresponding with diapause. Because their function is largely unknown, these proteins are usually referred to generically as "diapause-associated proteins."

The fat body tissues of pink bollworms entering diapause produce a characteristic protein that appears in the hemolymph during the wandering stage of the fourth- instar as a large molecular weight (490 kilodaltons) lipo-glyco-protein. The protein is not present in nondiapause larvae, never occurs in eggs or early larval instars and remains in high titre during diapause. The protein is reduced to low levels within 3 days of diapause termination. Thus, this pink bollworm protein fits the category of a diapause-associated protein. We have named it "pectinophorin" after the genus name of pink bollworm.

We devised a new method for measuring that protein in pink bollworm using an ELISA (enzyme-linked immunosorbant assay) test that is extremely sensitive to the presence of pectinophorin. The test was developed from polyclonal antibodies generated to pectinophorin; therefore, it is selective.

\section{ELISA test methods}

For the ELISA test, hemolymph was taken from a diapausing pink bollworm larva by removing a proleg and bleeding. Normally, $10 \mu \mathrm{l}$ were collected. This was diluted with water to $1 \mathrm{ml}$, and one-tenth of this solution (or $1 \mu \mathrm{l}$ of hemolymph) was dried overnight in the cold in a well of an ELISA plate. Any pectinophorin present stuck to the plastic ELISA plate material. After washing, the plates were

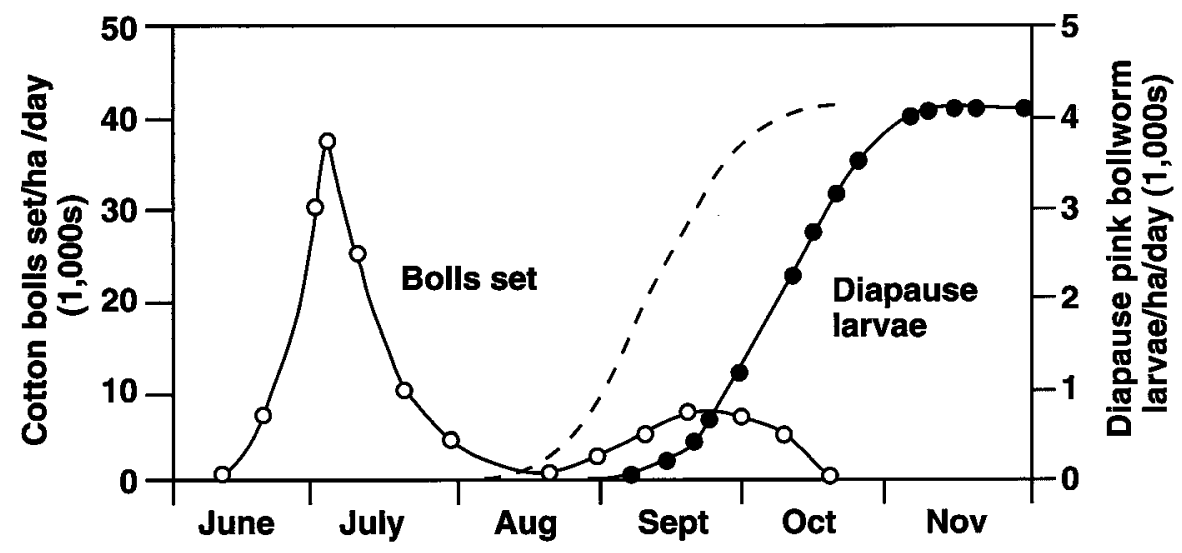

Fig. 1. Occurrence of diapause pink bollworm larvae (filled circles) and cotton bolls set (open circles) in Southern California and Arizona cotton fields. A suggested diapause curve (the dashed line) has been added to indicate what would be expected of a cotton field with heavy whitefly pressure. Modified from Kimball et al. (USDA publication, ARS-W49, 1977). 
flushed with bovine serum albu$\min$ (BSA) in phosphate-buffered saline (PBS). The BSA is said to block nonspecific sites, and experience shows that without this step, background readings are high.

Next, the primary antibody, antipectinophorin rabbit antibody, was added to the well. The antibody bound to any pectinophorin already in the well, and the remainder were washed off with phosphate-buffered saline plus Tween (PBST), a detergent.

After the antibody reaction, a goat antirabbit antibody that is conjugated to a color reagent, alkaline phosphatase, was added. The goat antibody bound to rabbit antibodies. Then, the ELISA plate was washed a second time and a substrate for the alkaline phosphatase, nitrophenylphosphate, was added. The product of reaction between the alkaline phosphatase and the nitrophenylphosphate, nitrophenol, is yellow in color. When the ELISA plate was placed in a spectrophotometer, the presence of yellow color was measured against a control, and densities of the products in the ELISA wells were obtained.

\section{ELISA test results}

Although pink bollworm populations are substantially lower than they have been in 25 years, as a result of the shortseason strategy, the Imperial Valley still has a low-level infestation. The new ELISA test results reveal that this residual population is probably coming from the small percentage of the population that is able to enter diapause in advance of the September 1 termination date.

On September 6, 1991, 1,600 cotton bolls were collected from the grounds of the University of Arizona Research Station on West 8th Street in Yuma and incubated in a screened area open to ambient conditions in El Centro. On September 9, fourthinstar crawlers were collected from beneath the incubation boxes and sent in a biomailer to Riverside.

Examination the next day at Riverside showed that the 110 pests collected in-

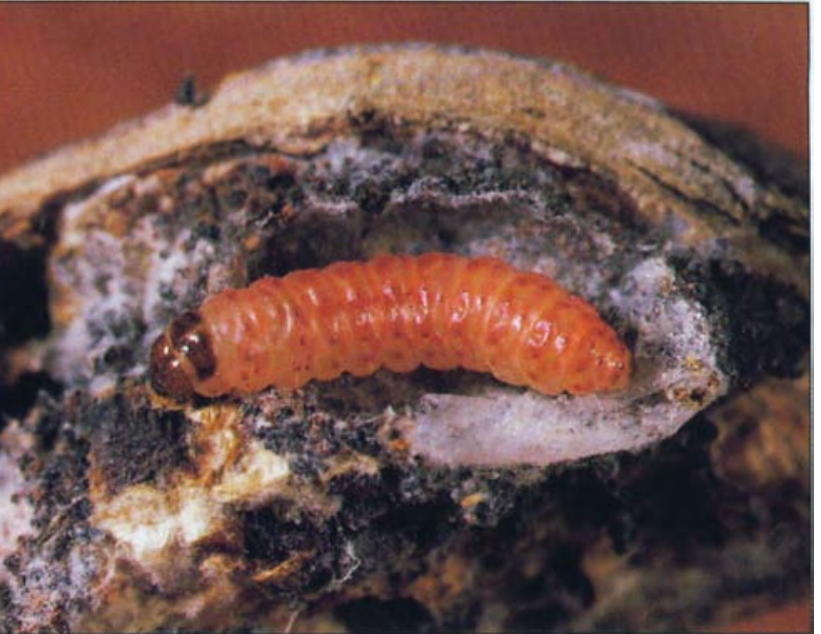

ELISA results with these (wells A-11, A-12 and B-11). Wells A$10, B-10$ and wells 10 and 11 of $C$ through $\mathrm{H}$ were all blanks to show background. Wells B-12

Mature pink bollworm larva. Photo by Jack Kelly Clark

cluded 19 larvae that were black and shriveled, obviously dead, and 9 pupae. These 28 dead and pupated pests were discarded. Ten of the remaining fourth-instar larvae were left undisturbed in their Petri dishes and placed in the laboratory at room temperature.

The 72 remaining larvae were prepared for the ELISA test immediately upon arrival as described above. The test subjects were bled on September 10 and the pectinophorin titres were obtained the next day. Table 1 gives the results.

For controls, we bled larvae kept in diapause in our laboratory and obtained through $\mathrm{H}-12$ were from active and feeding larvae that were obviously not in diapause and showed readings for pectinophorin well below threshold for diapause.

The blank or empty wells gave optical density (OD) readings of 0.136 or below with 0.119 the lowest value; 0.126 was the average density recorded. Density readings obtained from the active feeding larvae maintained in the laboratory ranged from 1.172 to 1.515 ; average value was 1.366. The larvae that were from our diapausing pests in the laboratory gave $O D$ values of 2.389 to 2.455 with an average of 2.422. Thus, there was a very clear difference between larvae in diapause and lar-

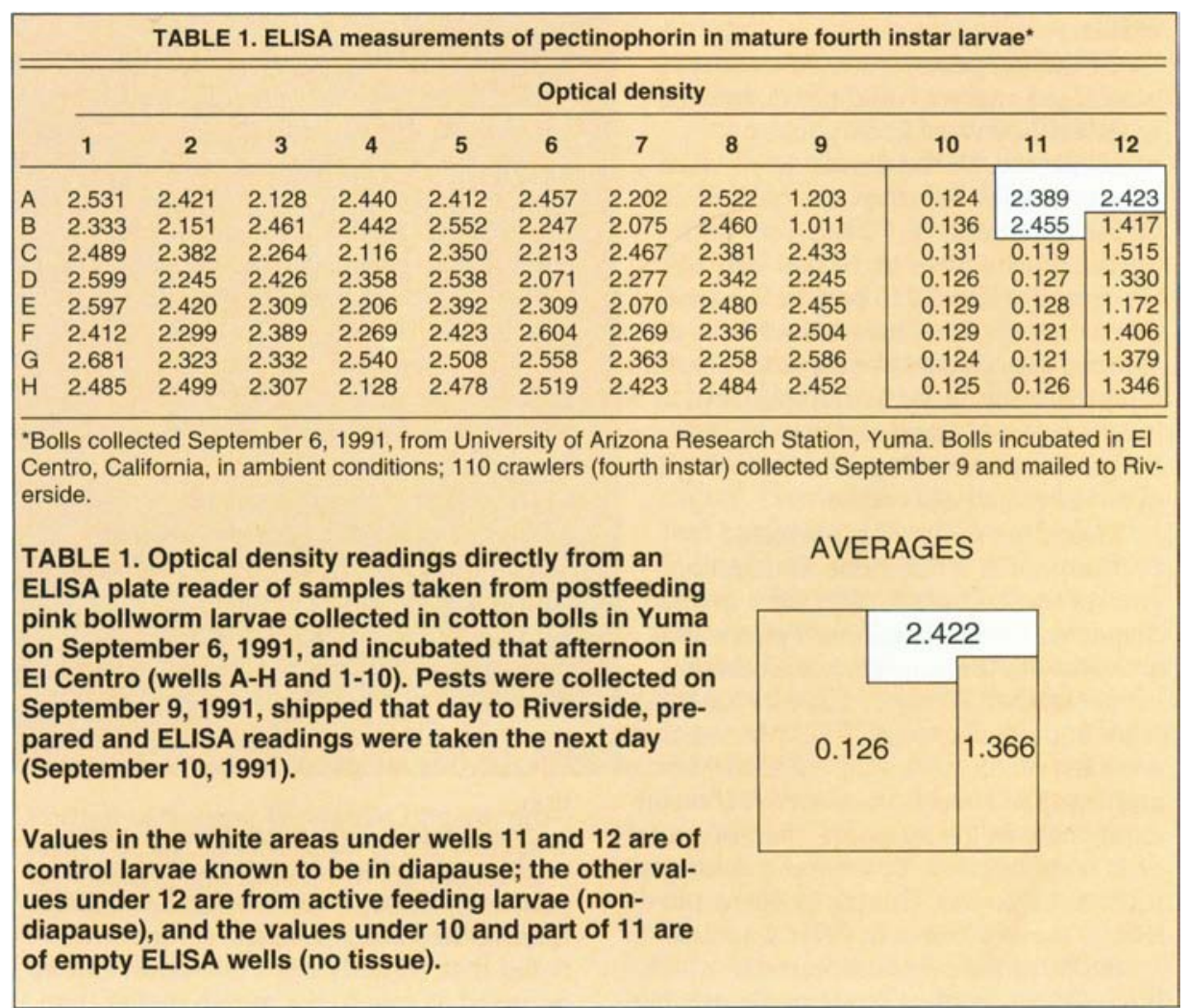




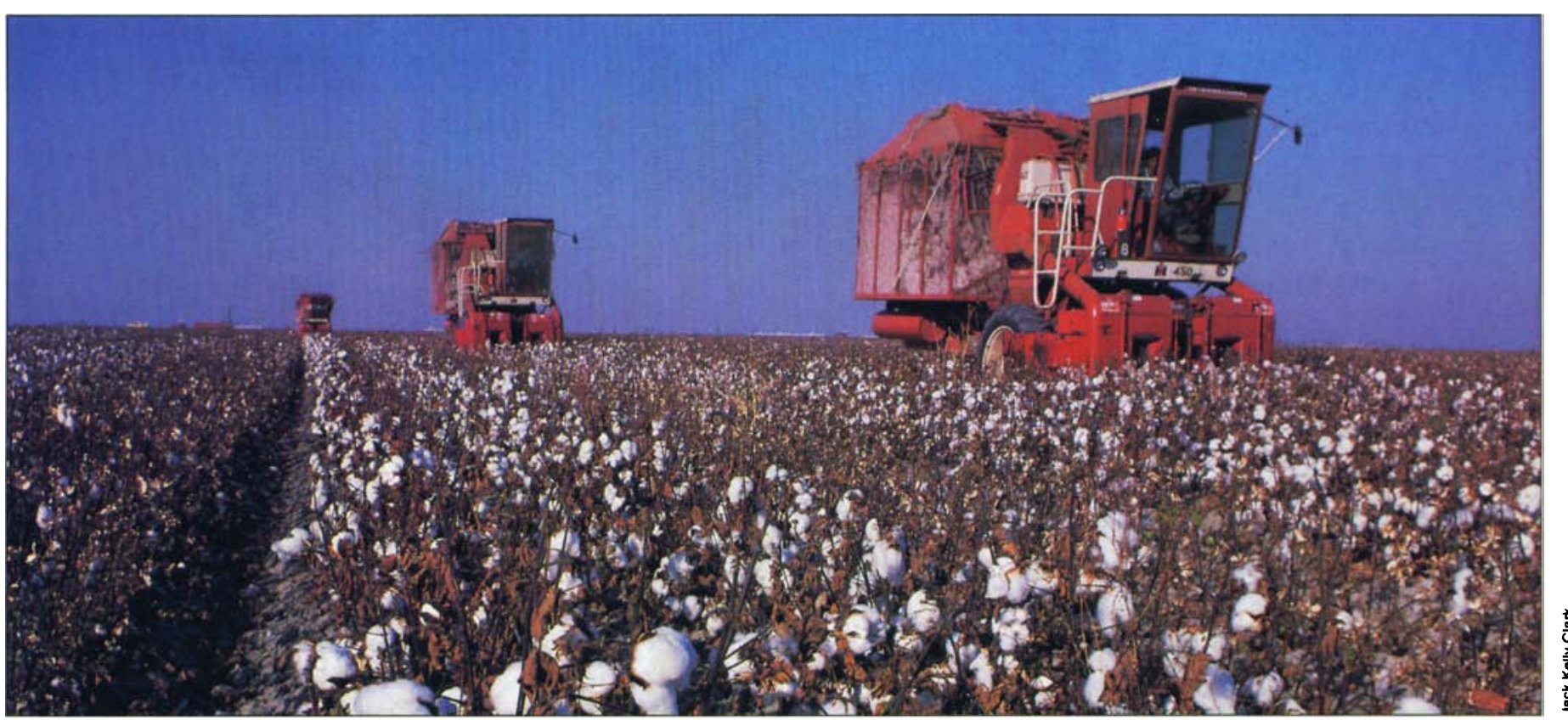

vae not in diapause in terms of presence of pectinophorin.

Of the 72 pests tested from those received, values fell either above a density of 2.070 with 2.681 as the maximum or they fell considerably below 2.000 . In fact, only two values were low, 1.203 and 1.011. The latter two had density values indistinguishable from blank or nondiapause controls. All of the other values ranged above and below the values obtained from larvae in deep diapause with high pectinophorin values.

Of the 110 pests sent to Riverside, 19 were dead and we could not determine whether they were in diapause or nondiapause. Of the remainder, 9 were pupae, or $9.9 \%$ nondiapause individuals. Of the remaining 72,70 had very high pectinophorin titres and 2 had very low. We consider these 2 to be nondiapause; if given enough time, they would have developed into pupae. We consider all of the others not only to be in diapause, but to be in an advanced stage of diapause because the pectinophorin titres were very high, not at intermediate values.

Therefore, of the 72 pests tested for pectinophorin, 2 had none. Put another way, of these 72 pests, $97 \%$ were in deep diapause. If we include the 9 pupae that arrived with the shipment with these numbers, then 70 out of 80 pests (or $88 \%$ ) were in deep diapause. The 10 larvae that were left on the bench top yielded 9 larvae and 1 pupae 1 week after arrival. If we include these in the numbers, then in a total of 91 testable pests, 12 were pupae or destined to be pupae. This gives the result that 79 (or $87 \%$ ) were in deep diapause.

If $87 \%$ of the pests that came from cotton bolls collected in Yuma on September
6 were entering diapause, then we can assume that the field pests had been entering diapause for at least the previous 2 weeks. It has been widely assumed that no diapausing larvae are collected on September 1 and up to $50 \%$ diapausing pests have been reported from collections on September 25 in normal years. (Figure 1 shows normally expected diapause curves from the literature as solid lines. Our results suggest a much earlier diapause, indicated as a dashed line.)

\section{Whitefly-induced early diapause}

To rationalize these results, first keep in mind that entrance into diapause is based not just on day length, but on temperature and the cotton plant's general nutritional condition as well. Larvae developing in the field are constantly undergoing temperature cycles since the temperature inside the cotton boll is about $2^{\circ} \mathrm{C}\left(4^{\circ} \mathrm{F}\right)$ below that of ambient. Therefore, the pink bollworm would be expected to be subject to the maximum and minimum swings of daily temperature.

A second element, recognized only recently, has been the adverse effect of whitefly (Bemisia tabaci) infestations on the cotton plant. During the 1991 growing season, many cotton plants were seen to defoliate in Southern California and Arizona, in some cases well in advance of fall. It was generally assumed then that the whitefly was responsible for the defoliation.

Such defoliation, regardless of cause, could explain the occurrence of early diapause in pink bollworm in 1991 and 1992. During the 1992 growing season, it was noted that whitefly-associated defoliation occurred as much as a month earlier than in the previous year. If defoliation events are occurring sooner, it becomes even more vital to accurately determine the onset of fall or late-summer diapause in pink bollworm, since a short-season strategy based on September 1 defoliation would allow significant diapause larvae to develop, depending on how much stress was induced on the cotton plants during the growing season. A suggested diapause curve was added to figure 1 as a dashed line to indicate what would be expected of a cotton field with heavy whitefly pressure.

An effort was mounted midway during the 1991 cotton-growing season by some growers and entomologists to delay the September termination date by 2 weeks (from September 1 to September 15), based largely on published accounts of the traditional measurements of diapause and on more recent repetitions of those measurements.

Results of the improved testing method reported here clearly show that overwintering populations not only would drastically increase if the termination date were delayed, but that the existing population will not go below a certain minimal number, even with continued use of the existing September 1 termination date.

T. A. Miller is Professor and M. Salama is postdoctoral researcher, Department of Entomology, UC Riverside, R. C. Weddle is Entomologist, Imperial County Agricultural Commissioner's Office, El Centro, and S. Sivasupramaniam is postdoctoral researcher, Department of Entomology, University of Arizona, Tucson.

Research reported here was supported entirely by funds from the California Cotton Pest Control Board. 